\title{
STEPANOFF FLOWS ON THE TORUS
}

\author{
JOHN C. OXTOBY
}

1. Let $X(x, y)$ and $Y(x, y)$ be of class $C^{\prime}$ in the $x y$-plane $M$ and periodic with period 1 in each variable. The equations

$$
\frac{d x}{d t}=X(x, y), \quad \frac{d y}{d t}=Y(x, y)
$$

define a flow $F_{M}$ in $M$ and also a flow $F_{\Omega}$ in the torus $\Omega$ obtained from $M$ by identifying $(x, y)$ with $\left(x^{\prime}, y^{\prime}\right)$ whenever $x-x^{\prime}$ and $y-y^{\prime}$ are integers. According to a classical theorem, these flows are area preserving if and only if

$$
\frac{\partial X}{\partial x}+\frac{\partial Y}{\partial y}=0
$$

A statement in a recent paper by Saito [6] suggested the following question: Does there exist on the torus an analytic, area preserving, ergodic flow which has a stationary point? The purpose of this note is to describe an elementary example of such a flow, and to discuss some properties of a topological class of flows to which it belongs.

Stepanoff [7] (cf. also [4, pp. 395-400, 506-507]) considered the flows defined by equations of the form (1) where $Y=\alpha X, \alpha$ is an irrational number, and $X$ is periodic, non-negative, continuous, satisfies a Lipschitz condition in $x$ and $y$, and vanishes at one and only one point of the torus. He showed that these flows are metrically transitive with respect to the invariant Borel measure

$$
\mu(E)=\iint_{E} \frac{d x d y}{X(x, y)} \quad(E \subset \Omega) .
$$

In general, $\mu(\Omega)$ may be either finite (e.g., $\left.X=\left(\sin ^{2} \pi x+\sin ^{2} \pi y\right)^{1 / 2}\right)$ or infinite (e.g., $X=\sin ^{2} \pi x+\sin ^{2} \pi y$ ). But if $X$ is required to be of class $C^{\prime}$, then $\mu(\Omega)=\infty$. Hence a flow of this kind will not serve to answer our question. Nevertheless, the example we are about to describe is in many respects similar.

2. Consider the flows $F_{M}$ and $F_{\Omega}$ defined by (1) where

$$
\begin{aligned}
& X=\alpha(1-\cos 2 \pi(x-y))+(1-\alpha)(1-\cos 2 \pi y), \\
& Y=\alpha(1-\cos 2 \pi(x-y))
\end{aligned}
$$

Received by the editors April 7, 1953. 
and where $\alpha$ is an irrational number between 0 and 1. Evidently $X$ and $Y$ are analytic, periodic, and satisfy (2). Hence the flows $F_{M}$ and $F_{\Omega}$ are analytic $[1$, p. 12] and area preserving. Moreover, $X=Y=0$ whenever both $x$ and $y$ are integers, and $X>0, Y \geqq 0$, elsewhere. In view of (2), the expression $-Y d x+X d y$ is an exact differential, which generates the integral function

$$
H=(1-\alpha)\left(y-\frac{\sin 2 \pi y}{2 \pi}\right)-\alpha\left(x-y-\frac{\sin 2 \pi(x-y)}{2 \pi}\right) .
$$

The orbits of $F_{M}$ are therefore contained in the curves of the family

$$
H(x, y)=c \quad(-\infty<c<\infty) .
$$

For each value of $c$, this equation determines $y$ as a single-valued function of $x$. The curve passes through the integral point $(n, m)$ if and only if $c=m-n \alpha$, hence no curve can pass through more than one such point. Because $X>0$ at all other points it follows that the orbits of $F_{M}$ are precisely the curves of the family (5), except that when $c=m-n \alpha$ the curve is composed of three orbits: the stationary point $(n, m)$, and the orbits of the two motions asymptotic to $(n, m)$ as $t$ tends to $+\infty$ and $-\infty$ respectively.

Each curve of the family (5) determines $y$ as an analytic function of $x$. This is clear at non-integral points, since there $H_{y}=X>0$. For the rest it is sufficient to consider the equation $H=0$. By taking a cube root this equation can be put in the form

$$
\beta y g(2 \pi y)=(x-y) g(2 \pi(x-y)),
$$

where $\beta=((1-\alpha) / \alpha)^{1 / 3}$ and $g(x)$ denotes the real cube root of $(x-\sin x) / x^{3}$, which is analytic and positive on the real axis. Equation (6) therefore defines a real analytic curve, whose slope at $(0,0)$ is $(1+\beta)^{-1}$. It should be noted that although each curve of the family (5) is analytic, the slope considered as a function of $x$ and $y$ is discontinuous at integral points. In fact, it assumes all values between 0 and 1 in every neighborhood of such a point. Closer inspection reveals that when $c$ is small but not zero the curve (5) exhibits a small "bump" as it passes near the origin, a behavior which might be anticipated since the origin is stationary and the flow incompressible.

In order to study this flow more conveniently, and in particular to determine all the finite invariant measures of $F_{\Omega}$, let us subject the plane $M$ to the transformation $S$ defined by

$$
x^{\prime}=x, \quad y^{\prime}=y+\frac{\alpha}{2 \pi} \sin 2 \pi(x-y)-\frac{1-\alpha}{2 \pi} \sin 2 \pi y .
$$


Each line parallel to the $y$-axis thereby undergoes a monotone increasing transformation into itself, hence $S$ is $1: 1$ as well as continuous. It follows (from invariance of region) that $S^{-1}$ is continuous. At nonintegral points $S^{-1}$ is indeed analytic, since there the Jacobian $(=X)$ of $S$ is different from zero, but at integral points $\partial y / \partial y^{\prime}$ becomes infinite.

Observe that if $S(x, y)=\left(x^{\prime}, y^{\prime}\right)$ then $S(x+n, y+m)=\left(x^{\prime}+n\right.$, $\left.y^{\prime}+m\right)$, hence $S$ also defines a topological transformation of $\Omega$ onto itself. Under $S, F_{M}$ and $F_{\Omega}$ are transformed into topologically equivalent flows, the curve $H(x, y)=c$ goes over into the straight line $y^{\prime}-\alpha x^{\prime}=c$, and the equations (1) are transformed into

$$
\frac{d x^{\prime}}{d t}=\Phi\left(x^{\prime}, y^{\prime}\right), \quad \frac{d y^{\prime}}{d t}=\alpha \Phi\left(x^{\prime}, y^{\prime}\right),
$$

where $\Phi\left(x^{\prime}, y^{\prime}\right)=X(x, y)$. This system is of the type considered by Stepanoff, except that $\Phi$ fails to satisfy a Lipshitz condition, in fact, $\Phi_{y^{\prime}}$ becomes infinite at integral points. However, this flow and those considered by Stepanoff belong to the slightly more general class we are about to consider.

3. By a Stepanoff flow we shall mean a one-parameter continuous group $F: P \rightarrow T_{t} P(-\infty<t<\infty)$ of transformations of the torus $\Omega$ onto itself such that (i) there is one and only one stationary point $P_{0}$, and (ii) the points of each orbit satisfy an equation of the form $y-\alpha x=$ constant, where $\alpha$ is an irrational number corresponding to F.

Note that (i) and (ii) imply that the orbits of $F$ coincide with the curves $y-\alpha x=$ constant, except that the curve through $P_{0}$ is the union of three orbits of $F$.

Two flows $P \rightarrow T_{t} P$ and $P \rightarrow S_{t} P$ in $\Omega$ are said to be topologically equivalent if there exists a homeomorphism $f$ of $\Omega$ onto itself such that $T_{t} f(P)=f\left(S_{t} P\right)$ for all $P$ and $t$.

Theorem 1. If a flow $F: P \rightarrow T_{t} P$ with stationary point $P_{0}$ is topologically equivalent to a Stepanoff flow, and if $F$ admits a normalized invariant Borel measure $\mu$ for which $\mu\left(P_{0}\right)=0$, then $\mu$ is unique and $F$ is ergodic with respect to $\mu$.

Proof. Since the properties in question are topologically invariant we may assume that $F$ is a Stepanoff flow and that $P_{0}$ corresponds to the origin in $M$. Suppose there exists an invariant Borel measure $\mu$ with $\mu(\Omega)=1$ and $\mu\left(P_{0}\right)=0$. Let $\Omega_{1}$ denote the complement of the three exceptional orbits in $\Omega$, then $\mu\left(\Omega_{1}\right)=1$. Let $C$ be the circle in $\Omega$ corresponding to the $y$-axis in $M$, and let $R$ denote the irrational 
rotation of $C$ corresponding to the translation $(0, y) \rightarrow(0, y+\alpha)$ of the $y$-axis. Put $\Gamma=C \cap \Omega_{1}$, then for each $P \in \Gamma$ there is a least positive number $t=\phi(P)$ such that $T_{t} P \in \Gamma$. Note that $\phi(P)$ is continuous on $\Gamma$, that it has a positive lower bound $2 h$, say, and that $T_{\phi(P)} P=R P$ on $\Gamma$. For each Borel set $B \subset \Gamma$ let

$$
B^{*}=\left\{T_{t} P: P \in B, 0<t \leqq h\right\} .
$$

Observe that the sets $B_{i}^{*}$ are disjoint whenever the sets $B_{i}$ are, and that $\mu\left(\Gamma^{*}\right)>0$, since $U_{n=-\infty}^{+\infty} T_{n h} \Gamma^{*}=\Omega_{1}$. Hence the formula

$$
m(B)=\frac{\mu\left(B^{*}\right)}{\mu\left(\Gamma^{*}\right)}
$$

defines a normalized Borel measure in $\Gamma$, and $m$ can be extended over $C$ by defining $m(C-\Gamma)=0$. To show that $m$ is invariant under $R$, take $0<\epsilon<h$ and put

$$
B_{i}=\{P: P \in B,(i-1) \epsilon<\phi(P) \leqq i \epsilon\} \quad(i=1,2, \cdots) .
$$

Then

$\mu\left(B^{*}\right)=\sum_{i=1}^{\infty} \mu\left(B_{i}^{*}\right)=\sum_{i=1}^{\infty} \mu\left(T_{i \epsilon} B_{i}^{*}\right) \leqq \mu\left(\left\{T_{t} P: P \in R B, 0<t<h+\epsilon\right\}\right)$.

Letting $\epsilon \rightarrow 0$ it follows that $m(B) \leqq m(R B)$. The reverse inequality is obtained by replacing $B$ by $\Gamma-B$. Since it is known that Lebesgue measure is the only normalized Borel measure in $C$ invariant under $R$, it follows that $R$ is ergodic with respect to $m$. If $E$ is any Borel subset of $\Omega$ invariant under $F$, let $B=E \cap \Gamma$ and $B^{\prime}=\Gamma-E$. Then $B$ and $B^{\prime}$ are invariant under $R$, hence $\mu\left(B^{*}\right) \mu\left(B^{\prime *}\right)=m(B) m\left(B^{\prime}\right)=0$, and since $B^{*}$ and $B^{\prime *}$ sweep out $E \cap \Omega_{1}$ and $\Omega_{1}-E$ respectively it follows that $\mu(E) \mu(\Omega-E)=0$, therefore $F$ is ergodic with respect to $\mu$. The uniqueness of $\mu$ follows from the observation that if there were two distinct ergodic measures in $\Omega_{1}$ their arithmetic mean would be nonergodic.

Theorem 1 implies that the flow $F_{\Omega}$ considered in $\$ 2$ is ergodic with respect to plane measure in $\Omega$, and that it has no other ergodic Borel measure except the trivial measure confined to the origin.

The following theorem shows that for any Stepanoff flow the space of normalized invariant measures (a convex subset of the space of bounded linear functionals on continuous functions on $\Omega$ ) consists either of a single point or of a segment joining two points.

We recall that a point $P$ is called quasi-regular [3] if the "time average $f^{*}(P)=\lim _{t \rightarrow \infty} f_{t}(P)$ exists for every continuous function $f$ on $\Omega$, where 


$$
f_{t}(P)=\frac{1}{t} \int_{0}^{t} f\left(T_{\tau} P\right) d \tau .
$$

Theorem 2. If a flow $F: P \rightarrow T{ }_{t} P$ with stationary point $P_{0}$ is topologically equivalent to a Stepanoff flow, then either (A) or (B) applies:

(A) The only normalized Borel measure invariant under $F$ is the trivial measure $\mu_{0}$ for which $\mu_{0}\left(P_{0}\right)=1$. In this case every point is quasiregular, and for any continuous function $f$ on $\Omega, f_{t}(P)$ converges uniformly on $\Omega$ to $f\left(P_{0}\right)$ as $t \rightarrow \infty$.

(B) $F$ has one and only one normalized ergodic Borel measure $\mu \neq \mu_{0}$, $\mu$ is the only invariant Borel measure for which $\mu(\Omega)=1$ and $\mu\left(P_{0}\right)=0$, and every finite invariant Borel measure is a linear combination of $\mu_{0}$ and $\mu$. In this case the quasi-regular points constitute a set of first category dense in $\Omega$.

Proof. If there is a unique normalized invariant Borel measure, then every point is quasi-regular, as was shown by Kryloff and Bogoliouboff [3, Theorem XV]. The uniformity of the convergence of $f_{t}(P)$ in this case has been shown for discrete flows in a previous paper $[5,(5.1)]$. The same reasoning applies here, and the limit is $\int f d \mu_{0}=f\left(P_{0}\right)$. In case there is a normalized invariant measure other than $\mu_{0}$ the first three assertions of (B) follow from Theorem 1, and it only remains to characterize the set $Q$ of quasi-regular points. Choose a continuous function $f$ and numbers $a$ and $b$ such that $f\left(P_{0}\right)<a<b<\int f d \mu$. Let

$$
\begin{aligned}
& E_{1}=\bigcap_{n=1}^{\infty} \bigcup_{t>n}\left\{P: f_{t}(P)<a\right\}, \\
& E_{2}=\bigcap_{n=1}^{\infty} \bigcup_{t>n}\left\{P: f_{t}(P)>b\right\} .
\end{aligned}
$$

Then $E_{1}$ and $E_{2}$ are $G_{\delta}$ sets. $E_{1}$ is dense since it contains the orbit of the motion that tends to $P_{0}$ as $t \rightarrow \infty . E_{2}$ is dense since it is invariant and since $\mu\left(E_{2}\right)=1$, by the ergodic theorem. Evidently $Q \subset \Omega$ $-\left(E_{1} \cap E_{2}\right)$, hence $Q$ is of first category. That $Q$ is dense follows from the fact that it is invariant and that $\mu(Q)=1[3$, Theorem II].

We remark that, in case (B), $\Omega$ is the orbit closure of a non-quasiregular point, since $Q$ is of first category and every orbit except $P_{0}$ is dense. Dowker [2] has shown, conversely, that $Q$ is of first category in any compact dynamical system in which the orbit of some point not in $Q$ is dense.

Theorem 2 generalizes results obtained by Stepanoff. Under the regularity conditions which he assumed a flow comes under case (B) 
whenever the measure defined by (3) is finite, and under case (A) otherwise. It should be noted that the last assertion of (A) implies that the time probability of sojourn of any point in any neighborhood of $P_{0}$ is equal to 1 , a property which Stepanoff established by direct calculation. Our reasoning shows in addition that the limit is approached uniformly with respect to $P$. Theorem 2 shows that in case (B) the set of all such points, other than $\boldsymbol{P}_{0}$, has invariant measure zero and is of first category. It would be interesting to know whether it includes anything except the orbit asymptotic to $\boldsymbol{P}_{\mathbf{0}}$.

It is easy to see that any Stepanoff flow is permanently regionally transitive, that is, for any two neighborhoods $U$ and $V$ there is a number $t_{0}$ such that $T_{t} U \cap V$ is nonempty for all $|t|>t_{0}$. In view of this and Theorem 1 it is natural to conjecture that every Stepanoff flow of type (B) is strongly mixing, but we have not been able to prove this.

\section{BIBLIOGRAPHY}

1. G. D. Birkhoff, Dynamical systems, New York, 1927.

2. Y. N. Dowker, The mean and transitive points of homeomorphisms, Ann. of Math. vol. 58 (1953) pp. 123-133.

3. N. Kryloff and N. Bogoliouboff, La theorie generale de la mesure dans son application d l'etude des systemes dynamiques de la mecanique non lineaire, Ann. of Math. vol. 38 (1937) pp. 65-113.

4. V. V. Nemyckil and V. V. Stepanov, Qualitative theory of differential equations, 2d ed., Moscow, 1949.

5. J. C. Oxtoby, Ergodic sets, Bull. Amer. Math. Soc. vol. 58 (1952) pp. 116-136.

6. T. Saito, On the measure-preserving flow on the torus, J. Math. Soc. Japan vol. 3 (1951) pp. 279-284, and vol. 4 (1952) p. 338.

7. W. Stepanoff, Sur une extension du theoreme ergodique, Compositio Math. vol. 3 (1936) pp. 239-253.

Bryn Mawr College 